\title{
Fast Microscale Acoustic Streaming Driven by a Temperature-Gradient-Induced Nondissipative Acoustic Body Force
}

Qiu, Wei; Joergensen, Jonas Helboe; Corato, Enrico; Bruus, Henrik; Augustsson, Per

Published in:

Physical Review Letters

Link to article, DOI:

10.1103/PhysRevLett.127.064501

Publication date:

2021

Document Version

Publisher's PDF, also known as Version of record

Link back to DTU Orbit

Citation (APA):

Qiu, W., Joergensen, J. H., Corato, E., Bruus, H., \& Augustsson, P. (2021). Fast Microscale Acoustic Streaming Driven by a Temperature-Gradient-Induced Nondissipative Acoustic Body Force. Physical Review Letters, 127(6), [064501]. https://doi.org/10.1103/PhysRevLett.127.064501

\section{General rights}

Copyright and moral rights for the publications made accessible in the public portal are retained by the authors and/or other copyright owners and it is a condition of accessing publications that users recognise and abide by the legal requirements associated with these rights.

- Users may download and print one copy of any publication from the public portal for the purpose of private study or research.

- You may not further distribute the material or use it for any profit-making activity or commercial gain

- You may freely distribute the URL identifying the publication in the public portal 


\title{
Fast Microscale Acoustic Streaming Driven by a Temperature-Gradient-Induced Nondissipative Acoustic Body Force
}

\author{
Wei Qiu $\odot,{ }^{1, *}$ Jonas Helboe Joergensen, ${ }^{2, \dagger}$ Enrico Corato, ${ }^{1}$ Henrik Bruus $\odot,{ }^{2, \ddagger}$ and Per Augustsson $\oplus^{1, \S}$ \\ ${ }^{1}$ Department of Biomedical Engineering, Lund University, Ole Römers väg 3, 22363 Lund, Sweden \\ ${ }^{2}$ Department of Physics, Technical University of Denmark, DTU Physics Building 309, DK-2800 Kongens Lyngby, Denmark
}

(Received 16 March 2021; accepted 30 June 2021; published 3 August 2021)

\begin{abstract}
We study acoustic streaming in liquids driven by a nondissipative acoustic body force created by lightinduced temperature gradients. This thermoacoustic streaming produces a velocity amplitude nearly 100 times higher than the boundary-driven Rayleigh streaming and the Rayleigh-Bénard convection at a temperature gradient of $10 \mathrm{~K} / \mathrm{mm}$ in the channel. The Rayleigh streaming is altered by the acoustic body force at a temperature gradient of only $0.5 \mathrm{~K} / \mathrm{mm}$. The thermoacoustic streaming allows for modular flow control and enhanced heat transfer at the microscale. Our study provides the groundwork for studying microscale acoustic streaming coupled with temperature fields.
\end{abstract}

DOI: 10.1103/PhysRevLett.127.064501

Acoustic streaming describes the steady time-averaged fluid motion that takes place when acoustic waves propagate in viscous fluids. The streaming flow is driven by a nonzero divergence in the time-averaged acoustic momentum-flux-density tensor [1]. Conventionally, in a homogeneous fluid, this nonzero divergence arises from two dissipation mechanisms of acoustic energy. The first case is the boundary-driven Rayleigh streaming [2], in which acoustic energy is dissipated in viscous boundary layers where the velocity of the oscillating fluid changes to match the surface velocity of the channel walls $[3,4]$ or of the suspended objects [5-8]. The resulting stress drives the flow [9], typically observed in standing wave fields in systems of a size comparable to the wavelength [10]. The second type of streaming, quartz wind or bulk-driven Eckart streaming [11], is driven by gradients induced by high acoustic wave attenuation typically associated with high-frequency traveling waves [11-13]. Both cases have been extensively studied, and the phenomenon of acoustic streaming continues to attract attention due to its importance related to medical ultrasound [14-17], thermoacoustic engines [18,19], acoustic levitation [7,20], manipulation of particles and cells in microscale acoustofluidics [21-28], and control of streaming by the shape of the walls $[29,30]$.

Recently, we discovered that boundary-driven streaming can be significantly suppressed in inhomogeneous media formed by solute molecules [31,32]. This suppression is attributed to the acoustic body force $f_{\text {ac }}$, which originates from the nonzero divergence in the time-averaged momentum-flux-density tensor induced by gradients in density and compressibility in the fluids $[33,34]$ and competes with the boundary-layer stress. The streaming rolls are confined to narrow regions near the channel walls, before the inhomogeneity is smeared out by diffusion and advection of the solute. This effect enables acoustic manipulation of submicrometer particles [35], such as bacteria [36], and trapping of hot plasma in gases [37].

In this Letter, we investigate microscale acoustic streaming in a liquid, in which the temperature-dependent compressibility and density have been made inhomogeneous by introducing a steady temperature gradient. We generate this gradient by light irradiation and absorption, and subsequently measure the streaming driven by the temperature-gradient-induced acoustic body force and call it thermoacoustic streaming. Using our newly developed model for thermovisocus acoustofluidics [38], the experimental results are validated and the mechanisms responsible for the thermoacoustic streaming are explained.

Our main findings are (i) the thermoacoustic streaming begins to disturb the boundary-driven Rayleigh streaming for a temperature gradient as small as $0.5 \mathrm{~K} / \mathrm{mm}$, resulting in streaming rolls with complex three-dimensional (3D) patterns. (ii) For a temperature gradient of $10 \mathrm{~K} / \mathrm{mm}$, the thermoacoustic streaming velocity is nearly 100 times higher than that of the boundary-driven Rayleigh streaming and of the Rayleigh-Bénard convection. (iii) In contrast to other types of acoustic streaming, the mechanism driving the thermoacoustic streaming is nondissipative.

The thermoacoustic streaming is of considerable fundamental relevance to a broad community of researchers working in nonlinear acoustics, thermoacoustics, microscale acoustofluidics, as well as heat transfer. For a microsystem, the advective streaming flow is remarkably high compared to the rate of thermal diffusion, and with a Péclet number $P e \approx 1$, heat transfer is strongly enhanced. Further, our findings pave the way for transient or steady control of the streaming through modulations of the temperature field or the acoustic field.

Experimental method.-The experiments were performed using a long straight microchannel of width 
$W=760 \mu \mathrm{m}$ and height $H=370 \mu \mathrm{m}$ in a glass-siliconglass chip with a piezoelectric transducer glued on. The temperature gradient inside the channel was generated by directing the focused light from a 470-nm light-emitting diode (LED) through water containing a dilute dye solution (0.1 wt \% Orange G) that has an overlapping absorption peak with the LED, see Fig. 1. We measured that $99 \%$ of the LED light was absorbed in the liquid. The transducer was run with an input power of $88 \mathrm{~mW}$ at $953 \mathrm{kHz}$, resulting in a standing half wave across the width with an acoustic energy density $E_{\mathrm{ac}}=9.24 \mathrm{~J} / \mathrm{m}^{3}$ measured as in Ref. [32]. The induced streaming was measured using the general defocusing particle tracking technique [39-41] at 5 to $60 \mathrm{fps}$ with $1.1 \mu \mathrm{m}$-diameter polystyrene tracer particles (red fluorescence), well below the critical diameter of $2.9 \mu \mathrm{m}$ that marks the cross-over to where streaming drag becomes the dominant force [35]. Green LED light passes through a band-pass filter $(525-550 \mathrm{~nm})$ and excites the tracer particles. It is barely absorbed by the dye and hence does not affect the temperature in the channel. The measurements under each condition were repeated 13 to 27 times and recorded in 7800 to 40500 frames to improve the statistics. The temperature field around the channel midheight plane was imaged using temperature-sensitive fluorescent dye (Rhodamine B); see the Supplemental Material [42].

Numerical model.-The model is the effective pressure acoustics model of Ref. [38], which includes thermoviscous

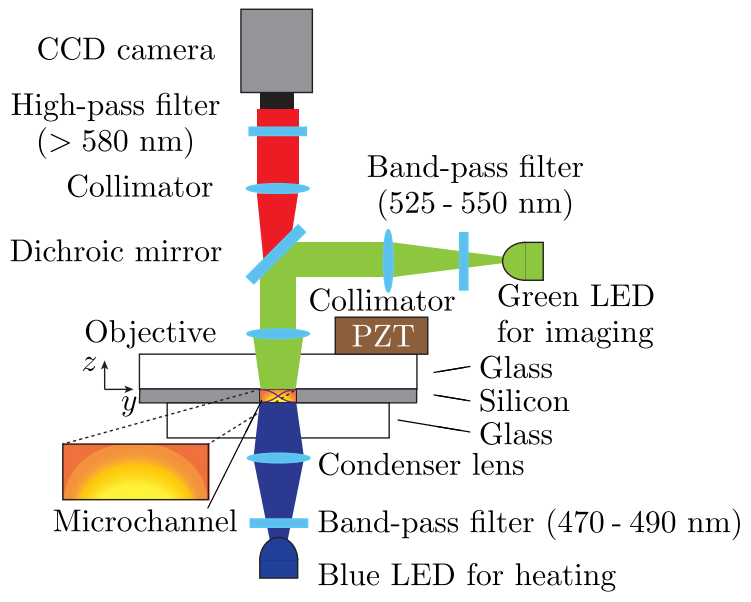

FIG. 1. Sketch of the acoustofluidic silicon chip (light gray) sandwiched between two glass layers (white) that allows optical transparency for both heating and particle tracking. The light emitted from a blue LED below the chip is absorbed by the aqueous dye solution and a temperature gradient forms from low (orange) to high (yellow) temperature in the channel. The piezoelectric transducer (PZT, dark brown) excites the resonant half-wave pressure field $p_{1}$ (purple) at $953 \mathrm{kHz}$. A green LED shines light from above to excite red fluorescent light from the tracer particles, which allows for optical recording of the tracer bead motion in a part of length $L=1300 \mu \mathrm{m}$ of the channel of width $W=760 \mu \mathrm{m}$ and height $H=370 \mu \mathrm{m}$. boundary layers and enables 3D simulations of thermoviscous acoustofluidic devices. To simulate the long glasssilicon-glass chip, symmetry planes are exploited to only simulate a quarter of the chip. Furthermore, the perfectly matched layer technique [53] is used to avoid simulating the entire length of the chip. The solver consists of three steps: (i) Computing the temperature field $T_{0}$ induced by the LED with an amplitude set to match the observed temperature gradients, (ii) computing the acoustic displacement $\boldsymbol{u}_{1}$ in the solid and the pressure $p_{1}$ in the fluid due to an actuation on the glass, and (iii) computing the resulting acoustic streaming field $v_{2}$. The heating from the LED is modeled with no absorption in the glass and an absorption parameter in the fluid selected to absorb $99 \%$ of the light passing through the chip as measured in the experiment. The model is based on perturbation theory, but the highest streaming velocities recorded in the experiments are found to be at the limit of the validity of the model, because there the thermoacoustic streaming begins to alter the temperature field $T_{0}$. For more details on the numerical model see the Supplemental Material [42]. Because the inherent difficulty in measuring the energy density $E_{\mathrm{ac}}$ at high streaming velocities when temperature gradients are present, the $E_{\mathrm{ac}}$ used in simulations is obtained by matching the experimental streaming velocity amplitude for each temperature gradient.

Results and discussion.-When both acoustics and temperature gradients are present, the streaming flow exhibits a complex 3D pattern. An example is shown in Figs. 2(a)-2(e), corresponding to a temperature difference $\Delta T_{0}=3.71 \mathrm{~K}$ across the channel of width $W=760 \mu \mathrm{m}$, equivalent to a gradient $G=2 \Delta T_{0} / W=9.76 \mathrm{~K} / \mathrm{mm}$. Here, two counterrotating deformed cylindrical streaming flow rolls appear, whirling with a velocity amplitude $\left|\boldsymbol{v}_{2}\right|=$ $1074 \mu \mathrm{m} / \mathrm{s}$ around the pair of curved white centerlines shown in Figs. 2(b) and 2(d). This velocity amplitude is about 77 and 87 times higher than that of the boundarydriven Rayleigh streaming and the Rayleigh-Bénard convection, respectively, under the same driving conditions (see Supplemental Material [42]).

The generation of this fast streaming can be explained by the acoustic body force $f_{\text {ac }}$ spawned by the temperature field induced by the blue LED. In this experiment, the light heats the fluid from beneath, while the silicon layer efficiently transports the heat away, thus cooling the sides of the channel. Temperature gradients are therefore induced in all directions: In the $x-y$ plane by the Gaussian profile of the light intensity and by the cooling from the silicon sidewalls, and in the $z$ direction by light absorption following the Beer-Lambert law. The resulting temperature field is highest at the center of the channel bottom, as shown by the measured and simulated temperature fields in the horizontal $x-y$ plane around channel mid-height $z=$ $0 \mu \mathrm{m}$ in Fig. 2(f), and by the simulated temperature field in the vertical $y-z$ cross section at $x=0 \mu \mathrm{m}$ in Fig. 2(g). The acoustic body force $f_{\text {ac }}$ depends on the gradients in 




FIG. 2. (a)-(e) The measured (exp, left half) and simulated (sim, right half) streaming flow for $G=9.76 \mathrm{~K} / \mathrm{mm}\left(\Delta T_{0}=3.71 \mathrm{~K}\right.$ across the channel width $W$ ), averaged in the indicated intervals (midposition \pm half-width) normal to the triplets of horizontal $x-y$ planes and vertical $x-z$ and $y-z$ planes. The vector plot (magenta) in a given plane is the in-plane velocity and the color plot is its magnitude from 0 (dark blue) to $1042 \mu \mathrm{m} / \mathrm{s}$ (yellow). The simulation is performed at $E_{\mathrm{ac}}=23 \mathrm{~J} / \mathrm{m}^{3}$. Spatial bins with no experimental data are excluded (gray). The pair of curved white lines in (b) and (d) represents the centerlines of the two counterrotating deformed cylindrical streaming flow rolls. The two line-plot insets show the measured (purple) and the simulated (green) $x$ (or $y$ ) component $v_{2}^{x}$ (or $v_{2}^{y}$ ) of the velocity along the red lines. (f) Color plot from $25.0^{\circ} \mathrm{C}$ (black) to $30.1^{\circ} \mathrm{C}$ (white) of the measured and simulated temperature $T_{0}$ in the horizontal $x-y$ plane around $z=0$; see more details in the Supplemental Material [42]. The regions where the fluorescence intensity is affected by the channel sidewalls are excluded (gray). (g) $T_{0}$ as in panel (f) but for the vertical $y-z$ plane at $x=0$; here, no experimental data are available.

compressibility and density, the acoustic pressure $p_{1}$, and the acoustic velocity $\boldsymbol{v}_{1}$ [33]. When the inhomogeneities are created by a temperature field, $f_{\text {ac }}$ can be expressed as,

$$
\begin{aligned}
\boldsymbol{f}_{\mathrm{ac}} & =-\frac{1}{4}\left|p_{1}\right|^{2} \nabla \kappa_{s, 0}-\frac{1}{4}\left|\boldsymbol{v}_{1}\right|^{2} \nabla \rho_{0} \\
& =-\frac{1}{4}\left[\left|p_{1}\right|^{2}\left(\frac{\partial \kappa_{s}}{\partial T}\right)_{T_{0}}+\left|\boldsymbol{v}_{1}\right|^{2}\left(\frac{\partial \rho}{\partial T}\right)_{T_{0}}\right] \boldsymbol{\nabla} T_{0} .
\end{aligned}
$$

Three factors determine the action of $\boldsymbol{f}_{\text {ac }}$ on the fluid. (i) Both the compressibility and density decrease with temperature, thus $\boldsymbol{f}_{\text {ac }}$ points towards the high temperature region, here the center of the channel heated by the LED. (ii) At room temperature, $\kappa_{s}\left|p_{1}\right|^{2} \approx \rho\left|\boldsymbol{v}_{1}\right|^{2}$ and $1 / \kappa_{s}\left|\partial_{T} \kappa_{s}\right| \gg 1 / \rho\left|\partial_{T} \rho\right|$, so $\boldsymbol{f}_{\text {ac }}$ is dominated by the $\left|p_{1}\right|^{2}$ compressibility term and thus is strongest at the pressure antinodes at the sides of the channel. (iii) As shown in
Fig. 2(g), the temperature gradient is larger at the bottom than at the top of the channel, resulting in a stronger $\boldsymbol{f}_{\mathrm{ac}}$ at the bottom. Consequently, in the bottom part of the LED spot, $f_{\text {ac }}$ pushes the fluid horizontally inward to the vertical $x-z$ center plane at $y=0$ and by mass conservation lets it escape outward along the axial $x$ direction and upward along the vertical $z$ direction. The resulting streaming flow contains the two aforementioned deformed cylindrical flow rolls, which when projected onto horizontal and vertical planes appear as the four horizontal streaming rolls in Figs. 2(a)-2(c) strongest in the center plane $z=0$, and as the two vertical streaming rolls in Fig. 2(d).

The thermoacoustic streaming is more than one order of magnitude faster than the boundary-driven Rayleigh streaming. It is mainly due to the nondissipative $f_{\text {ac }}$, a mechanism fundamentally different from the dissipation mechanism of the conventional forms of acoustic streaming. Moreover, the fast thermoacoustic streaming is stationary, because it is driven by the PZT transducer and the 

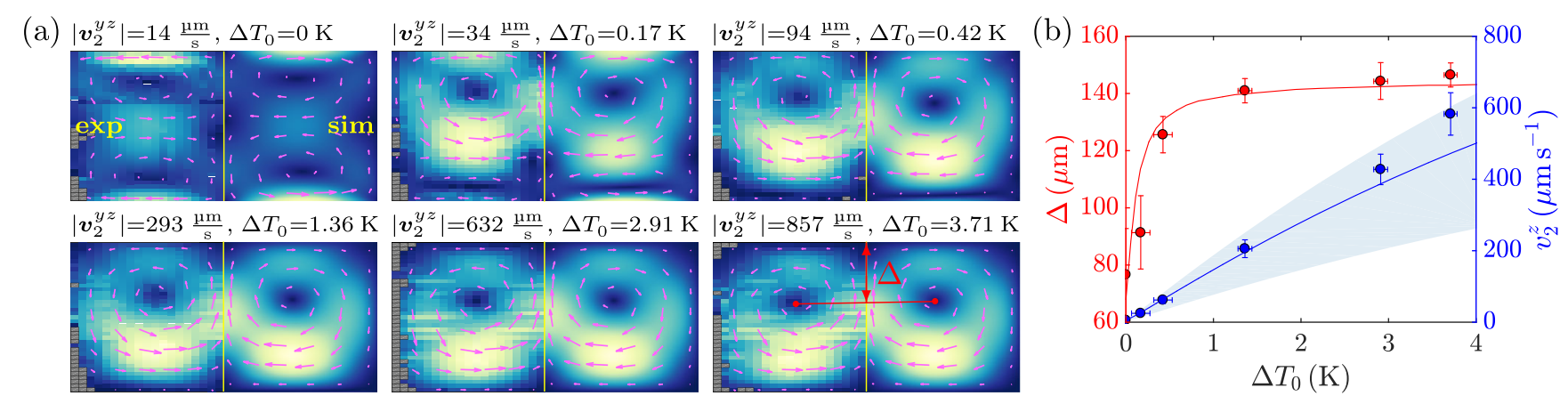

FIG. 3. (a) Measured (exp, left half) and simulated (sim, right half) in-plane streaming velocity $v_{2}^{y z}$ (magenta vectors) and its magnitude $\left|v_{2}^{y z}\right|$ from 0 (dark blue) to its maximum (yellow) in the vertical $y$-z plane averaged over the vertical slab $x=(0 \pm 100) \mu \mathrm{m}$ for six temperature differences across the channel ranging from $\Delta T_{0}=0$ to $3.71 \mathrm{~K}$. Also shown is the vortex size $\Delta$ (red). Simulations are performed with the energy density $E_{\text {ac }}$ for which $\left|v_{2}^{y z}\right|$ matches the experimental one under each $\Delta T_{0}$. Spatial bins with no experimental data points are excluded (gray). (b) Simulated (lines) and measured (points) vortex size $\Delta$ (red) and the $z$ component of the streaming velocity $\left|\boldsymbol{v}_{2}^{z}\right|$ (blue) versus $\Delta T_{0}$. The light blue region represents the simulated $\left|\boldsymbol{v}_{2}^{z}\right|$ for $E_{\mathrm{ac}}$ ranging from 9.24 (lower bound) to $23 \mathrm{~J} / \mathrm{m}^{3}$ (upper bound) with a reference (blue line) obtained at $E_{\text {ac }}=18 \mathrm{~J} / \mathrm{m}^{3}$.

LED light kept in steady state. In contrast, as shown in our previous studies [33,54], the streaming driven by an inhomogeneous distribution of solute molecules is unsteady, and it is fast only during the short initial transient advection-dominated relocation of solute molecules. In the subsequent long-lasting phase, where the solute concentration gradients are smeared out by diffusion, the streaming is strongly suppressed in the bulk $[31,32]$, and the boundary-driven streaming anywhere in this system is much slower than the fast thermoacoustic streaming.

The transition from boundary-driven Rayleigh streaming to thermoacoustic streaming is studied in the vertical $y-z$ cross section $(-100 \mu \mathrm{m}<x<100 \mu \mathrm{m})$ by gradually increasing the output power of the LED. Following Refs. [31,32], we quantify the streaming evolution by the vortex size $\Delta$, defined as the average of the distance from the center of each of the two upper flow rolls to the channel ceiling at $z=\frac{1}{2} H$. Figure 3 shows that for a zero temperature gradient $G=0$, the streaming is governed by the four conventional boundary-driven Rayleigh streaming rolls, whereas at a high gradient $G \approx 3.6 \mathrm{~K} / \mathrm{mm}$ $\left(\Delta T_{0}=1.36 \mathrm{~K}\right)$, only two big temperature-gradientinduced streaming rolls are present, driven by the relatively large $f_{\text {ac }}$ and occupying the whole channel cross section. In transitioning from the former to the latter situation, the two top Rayleigh flow rolls appear to expand downwards squeezing the bottom rolls against the channel floor at $z=-\frac{1}{2} H$. This phenomenon can be explained by the fact that the two top (bottom) Rayleigh flow rolls have the same (opposite) rotation direction as the two temperature-gradient-induced streaming rolls. Already at $G=0.5 \mathrm{~K} / \mathrm{mm}$ $\left(\Delta T_{0}=0.17 \mathrm{~K}\right), f_{\text {ac }}$ is large enough to distort the fourflow-roll Rayleigh streaming pattern. When $\Delta T_{0}$ further increases, the two-flow-roll thermoacoustic streaming pattern dominates, and eventually remains unchanged, while the velocity amplitude increases almost linearly for the investigated range, see Fig. 3(b).

The observation that thermoacoustic streaming occurs already at temperature gradients below $0.5 \mathrm{~K} / \mathrm{mm}$ calls for caution when combining acoustofluidic devices with optical systems. For an absorbing liquid, the light in a standard microscope is enough to induce strong velocity fields that may interfere with the study object. While we did not record the transient buildup of the streaming field upon activating the light, it can be noted that the development of the temperature field, and thus the streaming field, occurs within a few hundred milliseconds at the studied length scale, which enables rapid spatiotemporal modulation of local streaming fields through fast reconfigurable optical fields. Further, the induced streaming velocity is high enough to match the thermal diffusion time and thereby impact the heat transfer in the structure.

Conclusion.-This Letter describes a comprehensive experimental and numerical study of the thermoacoustic streaming in liquids induced by temperature gradients generated by light absorption in a microchannel. We have obtained a good match between measured and simulated velocity fields in three dimensions. As summarized by the main findings (i)-(iii) in the Introduction, the thermoacoustic streaming, driven by the nondissipative acoustic body force, has a markedly different origin than that of the conventional acoustic streaming associated with energy dissipation. Moreover, it reaches much higher velocity amplitudes compared to the boundary-driven Rayleigh streaming and the Rayleigh-Bénard convection under comparable conditions. The acoustic body force relies on the acoustic field and the gradients in compressibility and density, analogous to the driving force of the classical Rayleigh-Bénard convection relying on the gravitational field and the gradient in density. 
By including the temperature dependence of both density and compressibility in this work through our theory in Ref. [38], our analysis of thermoacoustic streaming in terms of the acoustic body force is valid for both liquids and gases. Thus, we have extended previous related work on gases, where compressibility effects are unimportant and therefore neglected [19,55-62].

This study is fundamental in scope, but also demonstrates a method with a clear potential for controlling local flows spatiotemporally in microchannels. Further, we highlight important implications of this phenomenon relating to heat transfer and integration of optical fields with microscale acoustofluidic devices.

We are grateful to R. Barnkob (Technical University of Munich) and M. Rossi (Technical University of Denmark) for providing the software DefocusTracker, and Masahiko Taniguchi (North Carolina State University) for the valuable information regarding dye properties. W. Q. was supported by the Foreign Postdoctoral Fellowship from Wenner-Gren Foundations and by MSCA EF Seal of Excellence IF-2018 from Vinnova, Sweden's Innovation Agency (Grant No. 2019-04856). This work has received funding from Independent Research Fund Denmark, Natural Sciences (Grant No. 8021-00310B) and the European Research Council (ERC) under the European Union's Horizon 2020 research and innovation programme (Grant Agreement No. 852590).

*wei.qiu@bme.lth.se

†jonashj@fysik.dtu.dk

*bruus@fysik.dtu.dk

§per.augustsson@bme.lth.se

[1] J. Lighthill, Acoustic streaming, J. Sound Vib. 61, 391 (1978).

[2] Lord Rayleigh, On the circulation of air observed in Kundt's tubes, and on some allied acoustical problems, Phil. Trans. R. Soc. London 175, 1 (1884).

[3] W. L. Nyborg, Acoustic streaming near a boundary, J. Acoust. Soc. Am. 30, 329 (1958).

[4] M. Hamilton, Y. Ilinskii, and E. Zabolotskaya, Acoustic streaming generated by standing waves in two-dimensional channels of arbitrary width, J. Acoust. Soc. Am. 113, 153 (2003).

[5] S. A. Elder, Cavitation microstreaming, J. Acoust. Soc. Am. 31, 54 (1959).

[6] C.P. Lee and T. G. Wang, Outer acoustic streaming, J. Acoust. Soc. Am. 88, 2367 (1990).

[7] A. Yarin, G. Brenn, O. Kastner, D. Rensink, and C. Tropea, Evaporation of acoustically levitated droplets, J. Fluid Mech. 399, 151 (1999).

[8] P. Tho, R. Manasseh, and A. Ooi, Cavitation microstreaming patterns in single and multiple bubble systems, J. Fluid Mech. 576, 191 (2007).

[9] H. Schlichting, Berechnung ebener periodischer Grenzeschichtströmungen, Phys. Z. 33, 327 (1932).
[10] P. B. Muller, M. Rossi, A. G. Marin, R. Barnkob, P. Augustsson, T. Laurell, C. J. Kähler, and H. Bruus, Ultrasound-induced acoustophoretic motion of microparticles in three dimensions, Phys. Rev. E 88, 023006 (2013).

[11] C. Eckart, Vortices and streams caused by sound waves, Phys. Rev. 73, 68 (1948).

[12] W. L. Nyborg, Acoustic streaming due to attenuated plane waves, J. Acoust. Soc. Am. 25, 68 (1953).

[13] N. Riley, Steady streaming, Annu. Rev. Fluid Mech. 33, 43 (2001).

[14] P. Marmottant and S. Hilgenfeldt, Controlled vesicle deformation and lysis by single oscillating bubbles, Nature (London) 423, 153 (2003).

[15] L. van der Sluis, M. Versluis, M. Wu, and P. Wesselink, Passive ultrasonic irrigation of the root canal: A review of the literature, Int. Endod. J. 40, 415 (2007).

[16] J. Wu and W. L. Nyborg, Ultrasound, cavitation bubbles and their interaction with cells, Adv. Drug Delivery Rev. 60, 1103 (2008).

[17] A. A. Doinikov and A. Bouakaz, Theoretical investigation of shear stress generated by a contrast microbubble on the cell membrane as a mechanism for sonoporation, J. Acoust. Soc. Am. 128, 11 (2010).

[18] H. Bailliet, V. Gusev, R. Raspet, and R. A. Hiller, Acoustic streaming in closed thermoacoustic devices, J. Acoust. Soc. Am. 110, 1808 (2001).

[19] M. Hamilton, Y. Ilinskii, and E. Zabolotskaya, Thermal effects on acoustic streaming in standing waves, J. Acoust. Soc. Am. 114, 3092 (2003).

[20] E. Trinh and J. Robey, Experimental study of streaming flows associated with ultrasonic levitators, Phys. Fluids 6, 3567 (1994).

[21] M. Wiklund, R. Green, and M. Ohlin, Acoustofluidics 14: Applications of acoustic streaming in microfluidic devices, Lab Chip 12, 2438 (2012).

[22] R. Barnkob, P. Augustsson, T. Laurell, and H. Bruus, Acoustic radiation- and streaming-induced microparticle velocities determined by microparticle image velocimetry in an ultrasound symmetry plane, Phys. Rev. E 86, 056307 (2012).

[23] B. Hammarström, T. Laurell, and J. Nilsson, Seed particle enabled acoustic trapping of bacteria and nanoparticles in continuous flow systems, Lab Chip 12, 4296 (2012).

[24] M. Antfolk, P. B. Muller, P. Augustsson, H. Bruus, and T. Laurell, Focusing of sub-micrometer particles and bacteria enabled by two-dimensional acoustophoresis, Lab Chip 14, 2791 (2014).

[25] A. Marin, M. Rossi, B. Rallabandi, C. Wang, S. Hilgenfeldt, and C. J. Kähler, Three-Dimensional Phenomena in Microbubble Acoustic Streaming, Phys. Rev. Applied 3, 041001 (R) (2015).

[26] P. Hahn, I. Leibacher, T. Baasch, and J. Dual, Numerical simulation of acoustofluidic manipulation by radiation forces and acoustic streaming for complex particles, Lab Chip 15, 4302 (2015).

[27] F. Guo, Z. Mao, Y. Chen, Z. Xie, J. P. Lata, P. Li, L. Ren, J. Liu, J. Yang, M. Dao, S. Suresh, and T. J. Huang, Threedimensional manipulation of single cells using surface acoustic waves, Proc. Natl. Acad. Sci. U.S.A. 113, 1522 (2016). 
[28] D. J. Collins, Z. Ma, J. Han, and Y. Ai, Continuous microvortex-based nanoparticle manipulation via focused surface acoustic waves, Lab Chip 17, 91 (2017).

[29] N. Nama, P.-H. Huang, T. J. Huang, and F. Costanzo, Investigation of acoustic streaming patterns around oscillating sharp edges, Lab Chip 14, 2824 (2014).

[30] J. S. Bach and H. Bruus, Suppression of Acoustic Streaming in Shape-Optimized Channels, Phys. Rev. Lett. 124, 214501 (2020).

[31] J. T. Karlsen, W. Qiu, P. Augustsson, and H. Bruus, Acoustic Streaming and Its Suppression in Inhomogeneous Fluids, Phys. Rev. Lett. 120, 054501 (2018).

[32] W. Qiu, J. T. Karlsen, H. Bruus, and P. Augustsson, Experimental Characterization of Acoustic Streaming in Gradients of Density and Compressibility, Phys. Rev. Applied 11, 024018 (2019).

[33] J. T. Karlsen, P. Augustsson, and H. Bruus, Acoustic Force Density Acting on Inhomogeneous Fluids in Acoustic Fields, Phys. Rev. Lett. 117, 114504 (2016).

[34] P. Augustsson, J. T. Karlsen, H.-W. Su, H. Bruus, and J. Voldman, Iso-acoustic focusing of cells for size-insensitive acousto-mechanical phenotyping, Nat. Commun. 7, 11556 (2016).

[35] W. Qiu, H. Bruus, and P. Augustsson, Particle-sizedependent acoustophoretic motion and depletion of micro-and nano-particles at long timescales, Phys. Rev. E 102, 013108 (2020).

[36] D. Van Assche, E. Reithuber, W. Qiu, T. Laurell, B. Henriques-Normark, P. Mellroth, P. Ohlsson, and P. Augustsson, Gradient acoustic focusing of sub-micron particles for separation of bacteria from blood lysate, Sci. Rep. 10, 3670 (2020).

[37] J. P. Koulakis, S. Pree, A. L. F. Thornton, and S. Putterman, Trapping of plasma enabled by pycnoclinic acoustic force, Phys. Rev. E 98, 043103 (2018).

[38] J. H. Joergensen and H. Bruus, Theory of pressure acoustics with thermoviscous boundary layers and streaming in elastic cavities, J. Acoust. Soc. Am. 149, 3599 (2021).

[39] R. Barnkob, C. J. Kähler, and M. Rossi, General defocusing particle tracking, Lab Chip 15, 3556 (2015).

[40] R. Barnkob and M. Rossi, General defocusing particle tracking: Fundamentals and uncertainty assessment, Exp. Fluids 61, 110 (2020).

[41] M. Rossi and R. Barnkob, A fast and robust algorithm for general defocusing particle tracking, Meas. Sci. Technol. 32, 014001 (2020).

[42] See Supplemental Material at http://link.aps.org/ supplemental/10.1103/PhysRevLett.127.064501 for details about the temperature measurements, the comparison between measured and simulated temperature fields, the numerical model, and the measured and simulated Rayleigh-Bénard convection, which includes Refs. [43-52].

[43] R.F. Kubin and A. N. Fletcher, Fluorescence quantum yields of some rhodamine dyes, J. Lumin. 27, 455 (1982).

[44] D. A. Hinckley, P. G. Seybold, and D. P. Borris, Solvatochromism and thermochromism of rhodamine solutions, Spectrochim. Acta A Mol. Spectrosc. 42, 747 (1986).

[45] J. Yoo, D. Mitchell, D. F. Davidson, and R. K. Hanson, Near-wall imaging using toluene-based planar laser-induced fluorescence in shock tube flow, Shock Waves 21, 523 (2011).

[46] B. Zhang, J. Zerubia, and J.-C. Olivo-Marin, Gaussian approximations of fluorescence microscope point-spread function models, Appl. Opt. 46, 1819 (2007).

[47] N. R. Skov, J. S. Bach, B. G. Winckelmann, and H. Bruus, 3D modeling of acoustofluidics in a liquid-filled cavity including streaming, viscous boundary layers, surrounding solids, and a piezoelectric transducer, AIMS Math. 4, 99 (2019).

[48] M. W. H. Ley and H. Bruus, Three-Dimensional Numerical Modeling of Acoustic Trapping in Glass Capillaries, Phys. Rev. Applied 8, 024020 (2017).

[49] J. Arnaud, W. Hubbard, G. Mandeville, B. De la Claviere, E. Franke, and J. Franke, Technique for fast measurement of gaussian laser beam parameters, Appl. Opt. 10, 2775 (1971).

[50] P. B. Muller, R. Barnkob, M. J. H. Jensen, and H. Bruus, A numerical study of microparticle acoustophoresis driven by acoustic radiation forces and streaming-induced drag forces, Lab Chip 12, 4617 (2012).

[51] A. Riaud, M. Baudoin, O. Bou Matar, J.-L. Thomas, and P. Brunet, On the influence of viscosity and caustics on acoustic streaming in sessile droplets: an experimental and a numerical study with a cost-effective method, J. Fluid Mech. 821, 384 (2017).

[52] J.S. Bach and H. Bruus, Theory of pressure acoustics with viscous boundary layers and streaming in curved elastic cavities, J. Acoust. Soc. Am. 144, 766 (2018).

[53] A. Bermúdez, L. Hervella-Nieto, A. Prieto, and R. Rodríguez, An optimal perfectly matched layer with unbounded absorbing function for time-harmonic acoustic scattering problems, J. Comput. Phys. 223, 469 (2007).

[54] J. T. Karlsen and H. Bruus, Acoustic Tweezing and Patterning of Concentration Fields in Microfluidics, Phys. Rev. Applied 7, 034017 (2017).

[55] M. K. Aktas and T. Ozgumus, The effects of acoustic streaming on thermal convection in an enclosure with differentially heated horizontal walls, Int. J. Heat Mass Transfer 53, 5289 (2010).

[56] G. Chini, Z. Malecha, and T. Dreeben, Large-amplitude acoustic streaming, J. Fluid Mech. 744, 329 (2014).

[57] M. Červenka and M. Bednařŕk, Effect of inhomogeneous temperature fields on acoustic streaming structures in resonators, J. Acoust. Soc. Am. 141, 4418 (2017).

[58] G. Michel and G. P. Chini, Strong wave-mean-flow coupling in baroclinic acoustic streaming, J. Fluid Mech. 858, 536 (2019).

[59] R. Fand and J. Kaye, Acoustic streaming near a heated cylinder, J. Acoust. Soc. Am. 32, 579 (1960).

[60] M. W. Thompson and A. A. Atchley, Simultaneous measurement of acoustic and streaming velocities in a standing wave using laser doppler anemometry, J. Acoust. Soc. Am. 117, 1828 (2005).

[61] M. W. Thompson, A. A. Atchley, and M. J. Maccarone, Influences of a temperature gradient and fluid inertia on acoustic streaming in a standing wave, J. Acoust. Soc. Am. 117, 1839 (2005).

[62] M. Nabavi, K. Siddiqui, and J. Dargahi, Effects of transverse temperature gradient on acoustic and streaming velocity fields in a resonant cavity, Appl. Phys. Lett. 93, 051902 (2008). 